\title{
Prevalence study of HPV mixed infections in Italian HIV positive women
}

\author{
Anna Rosa Garbuglia', Pierluca Piselli², Claudia Minosse', Catia Sias', Claudia Cimaglia², Alessandro Agresta², \\ Daniele Lapa', Franca Del Nonno³, Maria Rosaria Capobianchi' \\ I LaboratoriodiVirologia; \\ 2 Dipartimento Epidemiologia; \\ 3 Servizio Anatomia Patologica INMI L. Spallanzani, Roma
}

Key words: HPV, prevalence, HIV+ women

Studio di prevalenza di infezioni miste HPV in donne HIV positive in Italia

\section{SUMMARY}

Introduction: HIV positive women, show a higher frequency of multiple HPV infections than HIV negative. The immune response seems to be genotype-specific, but evidence on different genotypes distribution and involvement of coinfections in the development of invasive cervix cancer (ICC) remains limited. The aim of our study was to assess the prevalence of multiple infections in a group of Italian HIV positive women, the distribution of High risk (HR) strains and Low Risk (LR) strains in multiple and single infections, and their correlation with immune status and cervical lesions.

Methods: 553 women were considered in the study. HPV search was performed with MY09-MYII primers. HPV positive samples were typed with the Clinical Genomic array (HPV) test (Genomica, Spain).

Results: 244 samples were HPV positive (44.I\%).I29/244 (52.9\%) had a single infection and I03/244 (42.2\%) multiple infections. Among the $4 I 2$ performed typing, 223 (54.I\%) were HR strains, while I89 (45.9\%) were LR strains. The HPV6I (40 times) was more frequent among the LR strains. Among HR strains, the most frequently observed was the HPVI6 (30 times). In $92 \%$ of multiple infections, at least one HR strain was found. $36 \%$ of LR strains was presented in single infections compared to $27 \%$ of HR strains $(p=0.06)$.

The clades A3 ( $n=124,65.3 \%$ multiple infections) and AI0 ( $n=37,56.8 \%$ multiple infections) were the most represented in LR; $A 9$ ( $n=95$, $67.4 \%$ multiple infections) and $A 6(n=57,70.2 \%)$ clades were the most representative among HR strains. Differences in age between women with single infection and those with multiple infection were not observed $(p=0.33)$. Women with the best immune status (CD4 cell count of $>500$ cell $/ \mathrm{mm}^{3}$ ) showed a higher prevalence of single infection. HPV was positive in $75 \%$ of ASCUS/LSIL lesion and $77.3 \%$ of $\mathrm{H}-\mathrm{SIL}$.

Conclusions: HPV-I6 is the most frequent in both single and multiple infections as reported in a recent study about HIV negative women. Follow-up studies are necessary to assess the impact of multiple infections on the progression of different cervical lesion degrees.

\section{IINTRODUZIONE}

Approssimativamente 40 distinti genotipi Human papillomavirus (HPV) infettano il tratto cervico- genitale (Figura I) e almeno 14 di essi, definiti oncogenici o ad alto rischio (HR) sono associati con lo sviluppo del carcinoma invasivo della cervice (ICC). Donne ad alto rischio per lo sviluppo del tumore alla cervice, come le donne HIV positive, mostrano un'alta frequenza di avere infezioni multiple HPV rispetto alle donne HIV negative (3).

Uno stesso soggetto può essere infettato da più genotipi contemporaneamente. Studi epidemiologici suggeriscono che la risposta immune sembra essere genotipo-specifica (4), ma ancora le evidenze sulla distribuzione dei vari genotipi e la implicazione delle coinfezioni nello sviluppo dell'ICC rimangono limitate. L'obiettivo del nostro studio è stato quello di valutare la prevalenza delle infezioni multiple in un gruppo di donne italiane HIV positive, la eventuale associazione di specifici clades tra loro, la loro correlazione con lo stato immunitario e la lesione citologica.

\section{MATERIALI E METODI}

553 donne sono state seguite dal gennaio 2007 al giugno 2009 (età mediana 40.3 anni, range interquartile 35.145.8) presso l'INMI L. Spallanzani. La determinazione dell'HPV è stata eseguita con primers MY09-MY11 per una regione conservata dell'L1. Nei campioni HPV positivi si è proceduto alla tipizzazione con il kit Genomica che permette l'identificazione della presenza di più genotipi all'interno di un singolo campione. La diagnosi citologica è stata riportata usando il sistema Bethesda 2001. L'associazione tra variabili è stata analizzata per mezzo del test chi-quadro, mentre il confronto tra variabili continue è stato effettuato per mezzo del test non parametrico U di Mann-Whitney.

\section{RISULTATI}

Su 553 donne, 244 (44.1\%) sono risultate positive all'HPV (Figura II). Le donne HPV+ risultavano avere un'età e un numero di cellule CD4+ minore ( $\mathrm{p}<0.05) .129 / 244(52.9 \%)$ presentavano una infezione singola mentre 103/244 (42.2\%) presentavano una infezione multipla (di cui15 non ulteriormente tipizzabile) (Tabella 1).

Tra le 412 tipizzazioni effettuate, 223 (54.1\%) si riferivano a ceppi ad alto rischio (HR), mentre 189 (45.9\%) si riferivano a ceppi a basso rischio (LR). Come mostrato nella Figura III, tra i ceppi LR i più frequentemente osservati sono HPV61 (40 volte), HPV62 (26) e HPV6 (21); tra i ceppi HR, quelli più frequentemente osservati sono stati HPV16 (30 volte), HPV53 (29), HPV66 e 31 (22 ciascuno) (Figura III). Nel complesso su 244 donne risultate positive, 75 lo erano ad un ceppo LR (solo infezioni singole) mentre 142 ad uno o più ceppi HR (61 infezioni singole e 81 con infezioni multiple). Nel 92\% delle donne con infezioni multiple tipizzate è stato riscontrato almeno un ceppo HR, contro il 47.3\% riscontrato nelle donne con infezione singola $(\mathrm{p}<0.0001)$ (Tabella 1$)$.

Rispetto alle donne con infezione da ceppi a basso rischio, le donne positive ad uno o più ceppi HR risultavano avere un'età e un numero di cellule CD4+ minore $(\mathrm{p}<0.05)$; le donne con infezioni multiple risultavano avere un numero di cellule CD4+ minore $(\mathrm{p}<0.05)$, mentre relativamente all'età non presentavano una differenza statisticamente significativa rispetto alle donne HPV+ con infezioni singole ( $\mathrm{p}=0.33$ ). Il 36\% dei ceppi LR si presentavano in infezioni singole, contro il 27\% dei ceppi HR $(p=0.06)$. Tra gli HR il ceppo 53 era il più diffuso nelle infezioni singole, mentre nelle miste prevaleva il 16 . Tra i ceppi LR il 61 era il ceppo più rappresentato sia tra le infezioni singole che multiple. Le clades più rappresentate sono state la A3 ( $n=124,65.3 \%$ multiple) e A10 ( $n=37,56.8 \%$ multiple) tra i LR, mentre tra gli HR quelle più rappresentate

\section{Corresponding author: Anna Rosa Garbuglia}

Laboratorio di Virologia - INMI L. Spallanzani

00149 Roma - Via Portuense 292

E-mail: argarbuglia@iol.it 
sono state la A9 ( $n=95,67.4 \%$ multiple) e la A6 ( $n=57,70.2 \%$ multiple) (Tabella 2). Considerando l'associazione tra infezione da HPV e lesioni cervicali, l'infezione da HPV è stata riscontrata nel 75\% delle lesioni ASCUS/LSIL e nel 77.3\% delle lesioni di alto grado. In entrambi i casi lesioni cervicali si associano maggiormente con lesioni multiple (Tabelle 3 e 4).

\section{CONCLUSIONI}

La prevalenza delle infezioni multiple da HPV è un evento molto frequente nelle donne HIV positive. Tra i ceppi HR l'HPV16, come già riportato in altri studi $(1,5)$ risulta essere il più diffuso nelle infezione multiple. Questo dato può essere correlato alla maggiore capacità di persistenza dell'HPV16. La frequenza di infezioni multiple da HPV, la

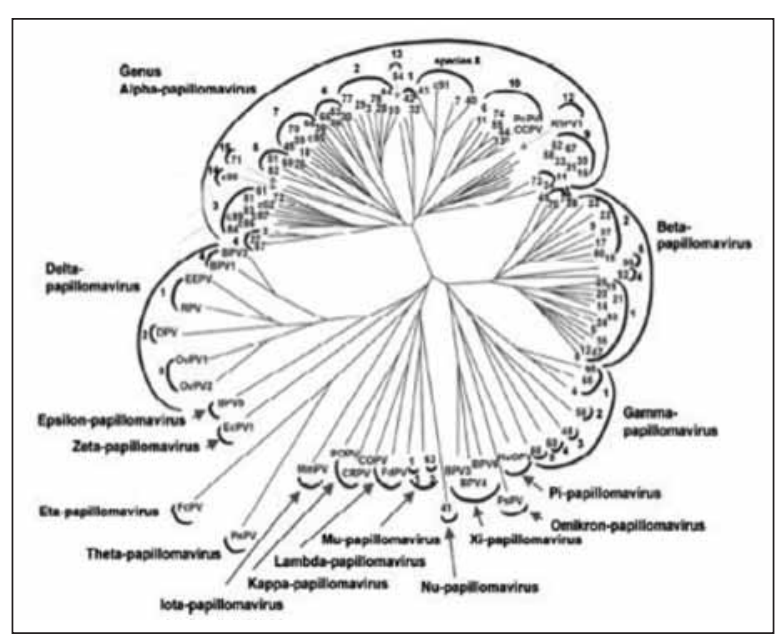

Figura I. Albero filogenetico contenente le sequenze di $1 / 8$ papillomavirus (5). loro distribuzione e l'associazione con il rischio di lesioni cervicali di basso ed alto grado sottolineano l'importanza di ricercare i singoli genotipi HPV per individuare eventuali fattori predittivi sull'outcome dell'infezione ed evidenziare potenziali limitazioni nelle attuali strategie di vaccinazione HPV.

L'associazione osservata tra i ceppi HR, soprattutto se presenti in infezioni multiple, e un numero di cellule CD4 positive minore potrebbe indicare una maggiore propensione nelle donne con uno stato di immunodeficienza più grave di presentare infezione da HPV, suggerendo un ruolo importante del sistema immunitario non solo del controllo dell'infezione da HPV ma anche della sua persistenza e molteplicità di infezione.

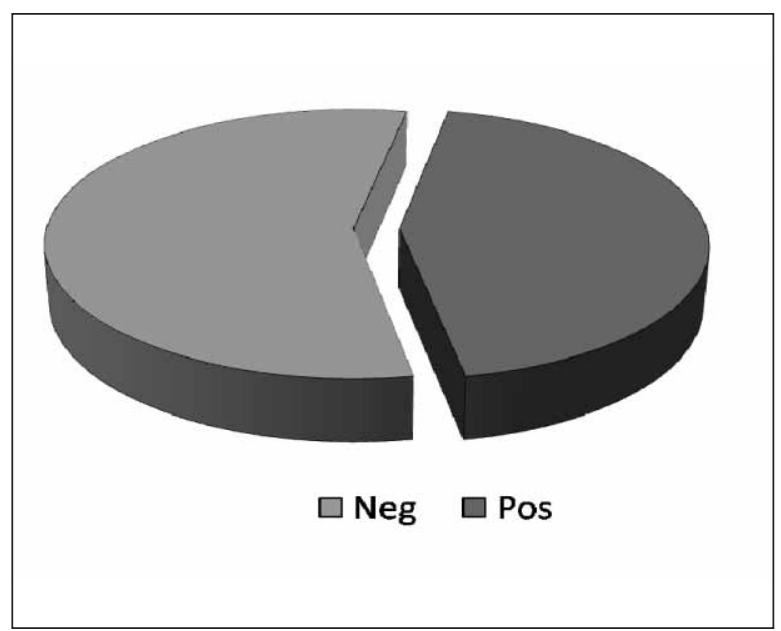

Figura II. Prevalenza infezione da HPV in 553 donne HIV Positive.

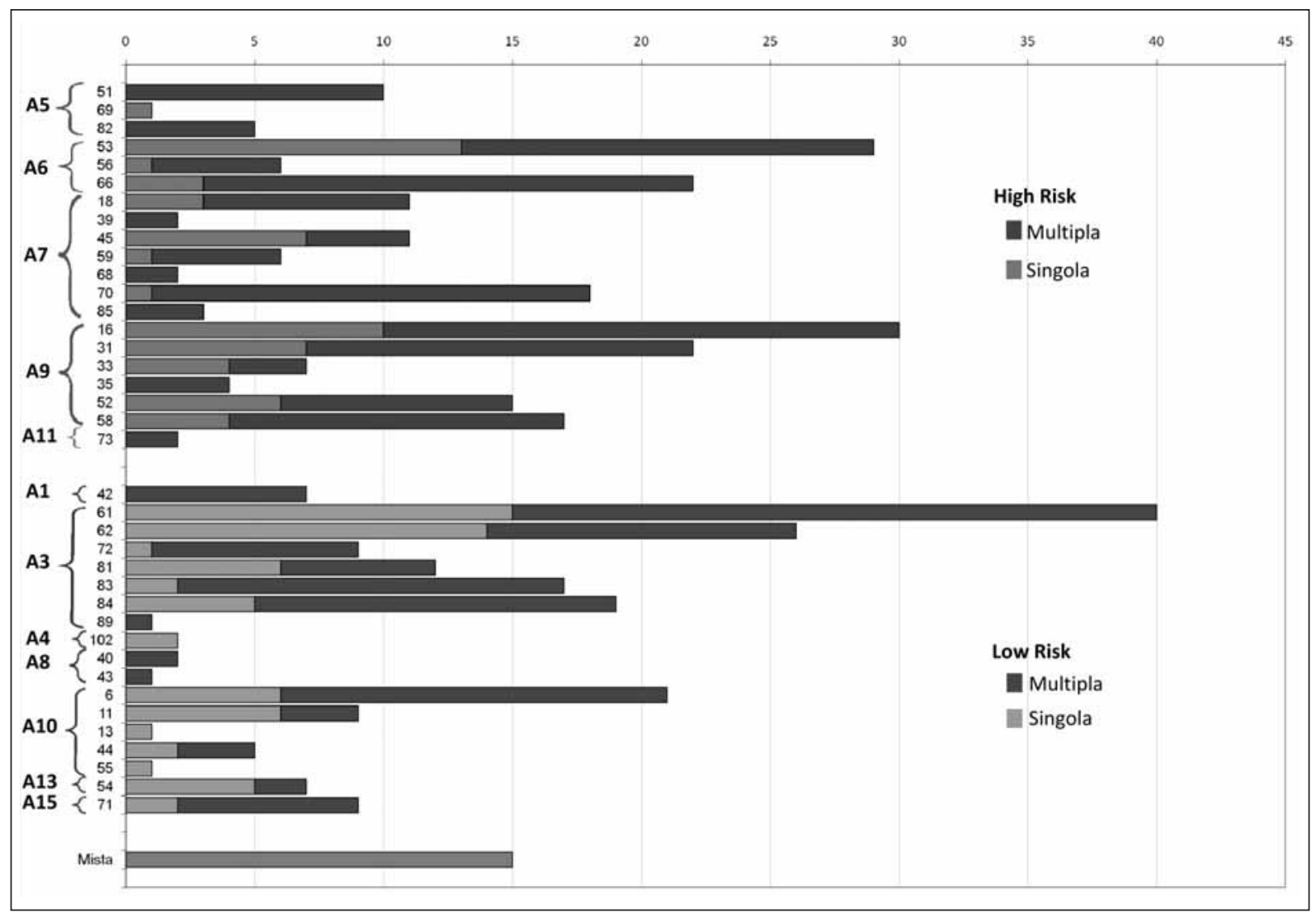

Figura III. Distribuzione dei genotipi di HPV in infezioni singole e multiple. 
Tabella I. Prevalenza delle infezioni singole e multiple in 217 donne HPV+ in base alla classificazione dei ceppi HPV.

\begin{tabular}{|c|c|c|c|c|c|c|}
\hline \multirow[b]{2}{*}{ Rischio } & \multicolumn{2}{|c|}{ SINGOLA } & \multicolumn{2}{|c|}{ MULTIPLA } & \multicolumn{2}{|c|}{ Totale } \\
\hline & $\mathbf{N}$ & $\%$ & $\mathbf{N}$ & $\%$ & $\mathbf{N}$ & $\%$ \\
\hline $\mathrm{HR}^{*}$ & 61 & 47.3 & 81 & 92.0 & 142 & 65.4 \\
\hline $\mathrm{LR}^{* *}$ & 68 & 52.7 & 7 & 8.0 & 75 & 34.6 \\
\hline Totale & 129 & 100.0 & 88 & 100.0 & 217 & 100.0 \\
\hline
\end{tabular}

*Almeno un ceppo HR; ** Almeno un ceppo LR

Tabella 2. Distribuzione dei genotipi di HPV in infezioni singole e multiple.

\section{CEPPI HIGH RISK (HR)}

\begin{tabular}{|c|c|c|c|c|}
\hline Clades & Tipo & Singola N (\%) & Multipla N (\%) & Tot \\
\hline \multirow[t]{3}{*}{$\overline{\mathrm{A5}}$} & 51 & $0(0)$ & $10(100)$ & 10 \\
\hline & 69 & $I(100)$ & $0(0)$ & $\mathrm{I}$ \\
\hline & 82 & $0(0)$ & $5(100)$ & 5 \\
\hline \multirow[t]{3}{*}{$\overline{A 6}$} & 53 & $13(44.8)$ & $16(55.2)$ & 29 \\
\hline & 56 & I (16.7) & $5(83.3)$ & 6 \\
\hline & 66 & $3(13.6)$ & $19(86.4)$ & 22 \\
\hline \multirow[t]{7}{*}{ A7 } & 18 & $3(27.3)$ & $8(72.7)$ & 11 \\
\hline & 39 & $0(0)$ & $2(100)$ & 2 \\
\hline & 45 & $7(63.6)$ & $4(36.4)$ & II \\
\hline & 59 & I (I6.7) & $5(83.3)$ & 6 \\
\hline & 68 & $0(0)$ & $2(100)$ & 2 \\
\hline & 70 & I (5.6) & $17(94.4)$ & 18 \\
\hline & 85 & $0(0)$ & $3(100)$ & 3 \\
\hline \multirow[t]{6}{*}{ A9 } & 16 & $10(33.3)$ & $20(66.7)$ & 30 \\
\hline & 31 & $7(3 \mid .8)$ & 15 (68.2) & 22 \\
\hline & 33 & $4(57.1)$ & $3(42.9)$ & 7 \\
\hline & 35 & $0(0)$ & $4(100)$ & 4 \\
\hline & 52 & $6(40)$ & $9(60)$ & 15 \\
\hline & 58 & $4(23.5)$ & $13(76.5)$ & 17 \\
\hline AlI & 73 & $0(0)$ & $2(100)$ & 2 \\
\hline Clades & & Singola N (\%) & Multipla N (\%) & Tot \\
\hline A5 & & I (6.3) & $15(93.8)$ & 16 \\
\hline A6 & & $17(29.8)$ & $40(70.2)$ & 57 \\
\hline A7 & & $12(22.6)$ & 4I (77.4) & 53 \\
\hline A9 & & $31(32.6)$ & 64 (67.4) & 95 \\
\hline AlI & & $0(0)$ & $2(100)$ & 2 \\
\hline
\end{tabular}

CEPPI LOW RISK (LR)

\begin{tabular}{|c|c|c|c|c|}
\hline Clades & Tipo & Singola N (\%) & Multipla N (\%) & Tot \\
\hline$\overline{A I}$ & 42 & $0(0)$ & $7(100)$ & 7 \\
\hline \multirow[t]{7}{*}{ A3 } & 61 & $15(37.5)$ & $25(62.5)$ & 40 \\
\hline & 62 & $14(53.8)$ & $12(46.2)$ & 26 \\
\hline & 72 & $I(I I . I)$ & $8(88.9)$ & 9 \\
\hline & 81 & $6(50)$ & $6(50)$ & 12 \\
\hline & 83 & $2(11.8)$ & $15(88.2)$ & 17 \\
\hline & 84 & $5(26.3)$ & $14(73.7)$ & 19 \\
\hline & 89 & $0(0)$ & $I(100)$ & $\mathrm{I}$ \\
\hline A4 & 102 & $2(100)$ & $0(0)$ & 2 \\
\hline \multirow[t]{2}{*}{$\overline{\mathbf{A 8}}$} & 40 & $0(0)$ & $2(100)$ & 2 \\
\hline & 43 & $0(0)$ & $I(100)$ & $\mathrm{I}$ \\
\hline \multirow[t]{5}{*}{ AIO } & 6 & $6(28.6)$ & I5 (7I.4) & 21 \\
\hline & 11 & $6(66.7)$ & $3(33.3)$ & 9 \\
\hline & 13 & $I(100)$ & $0(0)$ & $\mathrm{I}$ \\
\hline & 44 & $2(40)$ & $3(60)$ & 5 \\
\hline & 55 & $I(100)$ & $0(0)$ & $\mathrm{I}$ \\
\hline A/3 & 54 & $5(71.4)$ & $2(28.6)$ & 7 \\
\hline AI5 & 71 & $2(22.2)$ & $7(77.8)$ & 9 \\
\hline Clades & & Singola N (\%) & Multipla N (\%) & Tot \\
\hline Al & & $0(0)$ & $7(100)$ & 7 \\
\hline A3 & & $43(34.7)$ & $81(65.3)$ & 124 \\
\hline A4 & & $2(100)$ & $0(0)$ & 2 \\
\hline A8 & & $0(0)$ & $3(100)$ & 3 \\
\hline AlO & & $16(43.2)$ & $21(56.8)$ & 37 \\
\hline Al3 & & $5(7 \mid .4)$ & $2(28.6)$ & 7 \\
\hline Al5 & & $2(22.2)$ & $7(77.8)$ & 9 \\
\hline
\end{tabular}


Tabella 3. Associazione tra infezione da HPV e risultato del Pap-test

\begin{tabular}{|c|c|c|c|c|c|}
\hline & Neg & & Pos & & Totale \\
\hline PAPTEST & $\mathbf{N}$ & $\%$ & $\mathbf{N}$ & $\%$ & $\mathbf{N}$ \\
\hline NORMALE & 243 & 63.6 & 139 & 36.4 & 382 \\
\hline ASCUS/LSIL & 24 & 25.0 & 72 & 75.0 & 96 \\
\hline HSIL & 5 & 22.7 & 17 & 77.3 & 22 \\
\hline Totale & 272 & 54.7 & 228 & 45.3 & 500 \\
\hline
\end{tabular}

Tabella 4. Associazione tra infezioni singola e multipla e risultato del Pap-test

\begin{tabular}{|c|c|c|c|c|c|}
\hline \multirow[b]{2}{*}{ PAPTEST } & \multicolumn{2}{|c|}{ SINGOLA } & \multicolumn{2}{|c|}{ MULTIPLA } & \multirow[t]{2}{*}{ Totale } \\
\hline & $\mathbf{N}$ & $\%$ & $\mathbf{N}$ & $\%$ & \\
\hline NORMALE & 80 & 60.6 & 52 & 39.4 & 132 \\
\hline ASCUS/LSIL & 31 & 44.9 & 38 & 55.1 & 69 \\
\hline HSIL & 6 & 40.0 & 9 & 60.0 & 15 \\
\hline Totale & 117 & 54.2 & 99 & 45.8 & 216 \\
\hline
\end{tabular}

\section{BIBLIOGRAFIA}

1. Chaturvedi AK, Myers L, Hammons AF, et al. Prevalence and clustering patterns of human papillomavirus genotypes in multiple infections. Cancer Epidemiol Biomarkers Prev 2005; 14: 2439-45.

2. de Villers EM, Fauquet C, Broker TR, Bernard HU, zur Hausen H. Classification of papillomaviruses. Virology 2004; 324: 17-27.

3. Palefsky JM, Minkoff H, Kalish LA, et al. Cervicovaginal human papillo- mavirus infection in human immunodeficiency virus-1 (HIV)-positive and high-risk HIV-negative women. J Natl Cancer Inst 1999; 91: 226-36.

4. Roden RB, Greenstone HL, Kirnbauer R, et al. In vitro generation and type-specific neutralization of a human papillomavirus type 16 virion pseudotype. J Virol 1996; 70: 5875-83.

5. Spinillo A, Dal Bello B, Alberizzi P, et al. Clustering patterns of human papillomavirus genotypes in multiple infections. Virus Res 2009; 142:154-9. 